\title{
Long-wavelength dispersion of acoustic waves in transversely inhomogeneous anisotropic plates
}

\author{
A.L. Shuvalov*, O. Poncelet, M. Deschamps, C. Baron \\ Laboratoire de Mécanique Physique, Université Bordeaux 1, UMR CNRS 5469, 351 Cours de la Libération, \\ F-33405 Talence Cedex, France
}

Received 2 April 2004; received in revised form 3 March 2005; accepted 27 April 2005

Available online 24 June 2005

\begin{abstract}
Long-wavelength onset of the fundamental branches is described for a free anisotropic plate with arbitrary through-plate variation of material properties. Main attention is given to the flexural branch. Closed-form expressions for the leading-order dispersion coefficient of the velocity and displacement are derived for a generic case and exemplified for the various types of either continuous, or discrete, or periodic inhomogeneity and for the monoclinic symmetry. The relevance of the static averaging is examined in detail. The bounds for the slope of the flexural velocity branch are established. The upper fundamental branches are considered for the case when these are uncoupled inplane and shear horizontal ones.
\end{abstract}

(c) 2005 Elsevier B.V. All rights reserved.

Keywords: Inhomogeneous plate; Fundamental branches; Long-wave dispersion; Static averaging

\section{Introduction}

Acoustic-wave propagation in anisotropic transversely (vertically) stratified plates is closely involved with structural dynamics and non-destructive evaluation/testing of composites and, more recently, of functionally graded materials, which have broad area of applications [1-5]. Ultrasonics methods and NDE/NDT experiments are often carried out at relatively low frequency and deal with the onset of the fundamental dispersion branches. In this context, in general, and for efficient handling of the inverse problem in particular, it is helpful to know explicit analytical approximations of the long-wave dispersion. Such estimates are naturally tied in with the homogenization concept, see [6-8]. The objective of this paper is to obtain and simplify, insomuch as possible, the leading-order dispersion

\footnotetext{
* Corresponding author.

E-mail address: a.shuvalov@1mp.u-bordeaux1.fr (A.L. Shuvalov).
} 
coefficient at the onset of fundamental branches, to exemplify it for various types of inhomogeneity, and to relate its exact evaluation to the static averaging perspective.

Analytically straightforward access to the long-wave expansion of the fundamental velocity branches is provided by the sextic (state-vector) approach for plates. Its overview and the relevant bibliography for homogeneous and inhomogeneous plates is given in [9-11]. We shall therefore confine to the basic frame that suffices for the problem on hand (Section 2). Readily available explicit evaluation of the leading-order dispersion coefficient is yet obscured by multiple integrals. The flexural branch, however, allows a substantial simplification and detailed analysis even if anisotropy is arbitrary (Section 3). The upper fundamental branches are treated for the case of inplane/shear horizontal (SH) uncoupling, which allows relatively simple formulas for the SH fundamental branch (Section 4). A numerical example dealing with the set of fundamental branches in a continuously inhomogeneous plate is supplied (Section 5), followed by the conclusions (Section 6).

The basic algebraic notations used below are as follows: the superscript ' $T$ ' means transpose; $\otimes$ is the dyadic product; $\mathbf{I}$ and $\mathbf{I}_{(6)}$ are the $3 \times 3$ and $6 \times 6$ identity matrices; $\mathbf{T}$ is the $6 \times 6$ matrix with zero diagonal and identity off-diagonal $3 \times 3$ blocks.

\section{Background}

\subsection{Framework of the problem}

Consider an infinite plate with traction-free faces at $y= \pm H$ (the plate thickness is $2 H$ ), which consists of an arbitrary anisotropic and transversely inhomogeneous material with density $\rho(y)$ and elasticity tensor $\mathbf{c}(y)$. Inhomogeneity may be either continuous, or discrete-implying a stack of $n$ homogeneous layers, each with a thickness $2 H_{j}$ and constant $\rho^{(j)}$ and $\mathbf{c}^{(j)}(j=1,2, \ldots, n)$, or else it may combine ranges of continuous and discrete variation. Denote the unit vector along an arbitrary axis $X$ in the plane of plate faces by $\mathbf{m}$, and the unit vector along the axis $Y$ orthogonal to the faces by $\mathbf{n}$. Seeking the plane-wave solutions $\sim \exp [\mathrm{i} k(x-v t)]$ of the equation of motion leads, by way of the sextic formalism, to the system of six first-order ordinary differential equations with the matrix of coefficients $\mathbf{Q}(y)$. Its Stroh form, well-fitted with the long-wave expansion, is

$$
\mathbf{Q}=\mathrm{i} k\left(\begin{array}{cc}
\mathbf{N}_{1} & \mathbf{N}_{2} \\
\mathbf{N}_{3}-\rho v^{2} \mathbf{I} & \mathbf{N}_{1}^{\mathrm{T}}
\end{array}\right) ; \quad \mathbf{N}_{1}=-(n n)^{-1}(n m), \mathbf{N}_{2}=-(n n)^{-1}, \quad \mathbf{N}_{3}=(m m)-(m n)(n n)^{-1}(n m),
$$

where $(a b) \equiv a_{i} c_{i j k l} b_{l}$ for $\mathbf{a}, \mathbf{b}=\mathbf{m}$ or $\mathbf{n}$, and the components $c_{i j k l}$ of $\mathbf{c}(y)$ may be defined in a base $\left\{X_{1}, X_{2}, X_{3}\right\}$ of any orientation with respect to $X, Y$. The matricant solution referred to the plate faces, otherwise termed the propagator through the plate, is evaluated by the Peano expansion of the multiplicative integral of $\mathbf{Q}(y)$ [12],

$$
\begin{aligned}
\mathbf{M}(H,-H) & =\int_{-H}^{H}\left[\mathbf{I}_{(6)}+\mathbf{Q}(y) \mathrm{d} y\right]=\mathbf{I}_{(6)}+\int_{-H}^{H} \mathbf{Q}(y) \mathrm{d} y+\int_{-H}^{H} \int_{-H}^{y} \mathbf{Q}(y) \mathbf{Q}\left(y_{1}\right) \mathrm{d} y \mathrm{~d} y_{1}+\cdots \\
& =\mathbf{I}_{(6)}+\sum_{m=1}^{\infty}\left[\mathbf{P}^{(m)}\left(v^{2}\right)\right](2 \mathrm{i} k H)^{m} .
\end{aligned}
$$

Eq. (2) embraces the case of discrete inhomogeneity, when $\mathbf{M}(H,-H)=\prod_{j=n}^{1} \exp \left(2 H_{j} \mathbf{Q}_{\text {constant }}^{(j)}\right)$ and thus the multiple integrals of the Peano expansion reduce to multiple sums. The matrix coefficients $\mathbf{P}^{(m)}$ of the power series in (2) are the integrals or sums written, respectively, via the dimensionless variables

$$
\varsigma=\frac{y}{H}, \quad h_{j}=\frac{H_{j}}{H}
$$

which are used hereafter, while keeping the same notation for a function of $y$ and $\varsigma$ (e.g. $\mathbf{Q}(y) \Rightarrow \mathbf{Q}(\varsigma)$ ). 
The term of (2) with a single integral of $\mathbf{Q}$ implies the so-called static averaging, which will be denoted below for either continuous or discrete inhomogeneity by

$$
\langle\cdots\rangle=\left\{\begin{array}{l}
\frac{1}{2} \int_{-1}^{1} \cdots(\varsigma) \mathrm{d} \varsigma \\
\sum_{j=1}^{n} \ldots^{(j)} h_{j}
\end{array} .\right.
$$

For instance, the left off-diagonal block of $\langle\mathbf{Q}\rangle$ brings about $\langle\rho\rangle$ and $\left\langle\mathbf{N}_{3}\right\rangle$. The theory of the Stroh formalism [13] tells us that the symmetric matrix $\mathbf{N}_{3}$ has a null vector $\mathbf{n}$ and is positive definite in the subspace orthogonal to $\mathbf{n}$, hence the same is true for $\left\langle\mathbf{N}_{3}\right\rangle$ :

$$
\begin{aligned}
& \mathbf{N}_{3}(\varsigma) \mathbf{n}=0, \quad\left[\mathbf{N}_{3}(\varsigma)-\eta_{\alpha}(\varsigma) \mathbf{I}\right] \mathbf{e}_{\alpha}(\varsigma)=0\left(\text { or } \mathbf{N}_{3}^{(j)} \mathbf{n}=0,\left[\mathbf{N}_{3}^{(j)}-\eta_{\alpha}^{(j)} \mathbf{I}\right] \mathbf{e}_{\alpha}^{(j)}=0\right) ; \\
& \left\langle\mathbf{N}_{3}\right\rangle \mathbf{n}=0, \quad\left[\left\langle\mathbf{N}_{3}\right\rangle-\bar{\eta}_{\alpha} \mathbf{I}\right] \overline{\mathbf{e}}_{\alpha}=0 \quad(\alpha=2,3),
\end{aligned}
$$

where $\eta_{\alpha}(\varsigma)$ and $\bar{\eta}_{\alpha}>0$; the (unit) vectors $\mathbf{e}_{\alpha}(\varsigma)$ form an orthogonal triad with $\mathbf{n}$ and so do $\overline{\mathbf{e}}_{\alpha}$. Note that $\bar{\eta}_{\alpha}$ is certainly not an average of $\eta_{\alpha}(\varsigma)$ unless, due to the symmetry, $\mathbf{e}_{\alpha}(\varsigma)$ is constant and thus coincides with $\overline{\mathbf{e}}_{\alpha}$. That is, $\bar{\eta}_{\alpha}=\left\langle\eta_{\alpha}\right\rangle$ if and only if $\mathbf{e}_{\alpha}(\varsigma)=\overline{\mathbf{e}}_{\alpha}$ for any $\varsigma$ (or $\mathbf{e}_{\alpha}^{(j)}=\overline{\mathbf{e}}_{\alpha}$ for any $j$ ).

The boundary condition of traction-free plate faces demands vanishing determinant of the left off-diagonal block $\mathbf{M}_{3}$ of $\mathbf{M}(H,-H)$ and defines the displacement polarization at $y=-H$ as the null vector of $\mathbf{M}_{3}$. Inserting (2) into the dispersion equation det $\mathbf{M}_{3}=0$ enables explicit recursive finding of, in principle, any number of coefficients in the power-series expansions for the dispersion dependence of the three fundamental velocity branches. The series straighten if the viscosity is absent or is non-dispersive, for then the squared velocity $v_{\alpha}^{2}(k), \alpha=1,2,3$, expands into even powers of $2 k H$. It is clear that the limiting (zero-order) values at $k=0$ are determined by the eigenspectrum (5) of $\left\langle\mathbf{N}_{3}\right\rangle$ : the velocities are $v_{01}=0, v_{0 \alpha}=\sqrt{\bar{\eta}_{\alpha} /\langle\rho\rangle}$ and the polarizations are parallel to $\mathbf{n}$ and $\overline{\mathbf{e}}_{\alpha}$. The perturbationtheory procedure for obtaining the leading-order dispersion terms is the same as for homogeneous plates [9] to within the Peano expansion in place of the exponential of $\mathbf{Q}$. By this means the onset of the fundamental velocity branches is described as follows:

$$
\begin{aligned}
\langle\rho\rangle v_{1}^{2}= & (2 k H)^{2}\left[-\mathbf{n} \cdot \mathbf{P}_{3}^{(3)}(0) \mathbf{n}+\sum_{\alpha=2,3} \frac{1}{\bar{\eta}_{\alpha}}\left(\overline{\mathbf{e}}_{\alpha} \cdot \mathbf{P}_{3}^{(2)}(0) \mathbf{n}\right)\left(\mathbf{n} \cdot \mathbf{P}_{3}^{(2)}(0) \overline{\mathbf{e}}_{\alpha}\right)\right] ; \\
\langle\rho\rangle v_{\alpha}^{2}= & \bar{\eta}_{\alpha}-(2 k H)^{2}\left[\overline{\mathbf{e}}_{\alpha} \cdot \mathbf{P}_{3}^{(3)}\left(v_{0 \alpha}^{2}\right) \overline{\mathbf{e}}_{\alpha}+\frac{1}{\bar{\eta}_{\alpha}}\left(\overline{\mathbf{e}}_{\alpha} \cdot \mathbf{P}_{3}^{(2)}\left(v_{0 \alpha}^{2}\right) \mathbf{n}\right)\left(\mathbf{n} \cdot \mathbf{P}_{3}^{(2)}\left(v_{0 \alpha}^{2}\right) \overline{\mathbf{e}}_{\alpha}\right)\right. \\
& \left.+\frac{1}{\bar{\eta}_{\alpha}-\bar{\eta}_{\beta}}\left(\overline{\mathbf{e}}_{\alpha} \cdot \mathbf{P}_{3}^{(2)}\left(v_{0 \alpha}^{2}\right) \overline{\mathbf{e}}_{\beta}\right)\left(\overline{\mathbf{e}}_{\beta} \cdot \mathbf{P}_{3}^{(2)}\left(v_{0 \alpha}^{2}\right) \overline{\mathbf{e}}_{\alpha}\right)\right], \quad \alpha, \beta=2,3(\alpha \neq \beta),
\end{aligned}
$$

where $\mathbf{P}_{3}^{(m)}$, the left off-diagonal blocks of $\mathbf{P}^{(m)}$ in (2), are

$$
\begin{aligned}
\mathbf{P}_{3}^{(2)}\left(v^{2}\right)= & \frac{1}{2^{2}} \int_{-1}^{1} \int_{-1}^{\varsigma}\left[\mathbf{N}_{3}\left(v^{2}, \varsigma\right) \mathbf{N}_{1}\left(\varsigma_{1}\right)+\mathbf{N}_{1}^{\mathrm{T}}(\varsigma) \mathbf{N}_{3}\left(v^{2}, \varsigma_{1}\right)\right] \mathrm{d} \varsigma \mathrm{d} \varsigma_{1} \\
\mathbf{P}_{3}^{(3)}\left(v^{2}\right)= & \frac{1}{2^{3}} \int_{-1}^{1} \int_{-1}^{\varsigma} \int_{-1}^{\varsigma_{1}}\left[\mathbf{N}_{3}\left(v^{2}, \varsigma\right) \mathbf{N}_{1}\left(\varsigma_{1}\right) \mathbf{N}_{1}\left(\varsigma_{2}\right)+\mathbf{N}_{1}^{\mathrm{T}}(\varsigma) \mathbf{N}_{3}\left(v^{2}, \varsigma_{1}\right) \mathbf{N}_{1}\left(\varsigma_{2}\right)\right. \\
& \left.+\mathbf{N}_{3}\left(v^{2}, \varsigma\right) \mathbf{N}_{2}\left(\varsigma_{1}\right) \mathbf{N}_{3}\left(v^{2}, \varsigma_{2}\right)+\mathbf{N}_{1}^{\mathrm{T}}(\varsigma) \mathbf{N}_{1}^{\mathrm{T}}\left(\varsigma_{1}\right) \mathbf{N}_{3}\left(v^{2}, \varsigma_{2}\right)\right] \mathrm{d} \varsigma \mathrm{d} \varsigma_{1} \mathrm{~d} \varsigma_{2},
\end{aligned}
$$

with $\mathbf{N}_{3}\left(v^{2}, \varsigma\right)=\mathbf{N}_{3}(\varsigma)-\rho(\varsigma) v^{2} \mathbf{I}$. 
The purpose of the following consideration is simplification and analysis of the leading-order dispersion coefficients, encompassed by (6)-(8). The case of (non-dispersive) viscosity, admitted above, is not pursued. The higher order coefficients grow lengthy and therefore will be presented only for the case of $\mathrm{SH}_{0}$ fundamental branch.

\subsection{Preliminary remarks}

Let us accentuate in advance some evident, yet noteworthy points of the upcoming derivations. Firstly, it is reminded that Eqs. (6)-(8) represent truncated series (in powers of $2 \mathrm{kH}$ ) of the exact solution for the fundamental wave velocity and thus we are dealing with an exact value of the leading-order dispersion coefficient. Secondly, it is noted that adhering to the long-wave approximation does not indeed restrict the impact of inhomogeneity, i.e. the condition $2 k H \ll 1$ does not imply weak inhomogeneity $\Delta \sim 2 H \mathbf{N}^{\prime} /\langle\mathbf{N}\rangle \ll 1$ (where $\mathbf{N}^{\prime}$ is a 'typical value' of derivative in $y$ ) and so the coefficients of the series, including the leading-order one, may be anyhow strongly affected by the variation of material properties through the plate. Thirdly, treating the long-wave range, it is natural to establish a link to the concept of statically homogenized, or 'effective' medium, which is defined as the non-dispersive homogeneous material with the density $\langle\rho\rangle$ and elasticity tensor $\mathbf{c}_{\text {eff }}(\neq\langle\mathbf{c}\rangle)$ inferred from the statically averaged matrix $\langle\mathbf{N}\rangle$. Obviously, the zero-order term of the series is supplied by statically averaged properties of an inhomogeneous plate, which are inserted into the formula for a homogeneous plate (i.e. $\rho v_{0 \alpha}^{2}=0, \bar{\eta}_{2}(\varsigma), \bar{\eta}_{3}(\varsigma)$ with eigenvalues of $\left\langle\mathbf{N}_{3}\right\rangle$ in place of that of $\mathbf{N}_{3}=$ constant). Equally evident is that the same course of action - using 'homogeneous formulas' with statically averaged parameters - would be incorrect for exact evaluation of the leading-order dispersion coefficients. Indeed, their calculation, according to (7), involves the matricant expansion (2) up to the $(2 k H)^{3}$-order, whereas the static averaging is equivalent to truncating this series by the linear term in $2 \mathrm{kH}$. At the same time it is clear that the static averaging can certainly provide an approximation if the inhomogeneity is weak $(\Delta \ll 1)$ and also if its variation within the plate is described by, loosely speaking, a rapidly oscillating function with a slowly changing envelope, in particular if it is the case of a periodic plate with large enough number of periods (the reservation concerning the $\mathrm{SH}_{0}$ branch is in order, see Section 4). Barring those plain setups, the question in general is what is the relation, if any, between the true value of the leading-order dispersion coefficient and the static averaging benchmark. Regarding the flexural branch, we shall see that the bound(s) for former value can be expressed via the latter, and that the difference between these two can be used for a qualitative insight into the local features of certain types of the inhomogeneity profile. To this end it is noted, that, whereas the static averaging involves only the integral inhomogeneity, calculation of the true dispersion coefficient deals with the momentums of elastic inhomogeneity profile.

\section{Flexural branch}

\subsection{Arbitrary anisotropy}

Denote the leading-order coefficient in the expansion (6) by $\chi$ and the slope of the flexural branch $v_{1}(k)$ at its origin point by $\kappa$ (separate notations will prove handy), so that the truncated dispersion relation reads

$$
\langle\rho\rangle v_{1}^{2}(k)=\chi(2 k H)^{2} \quad \text { or } \quad v_{1}(k)=2 \kappa k H \quad\left(\text { or } v_{1}(\omega)=\sqrt{2 \kappa \omega H}\right), \text { where } \kappa=\sqrt{\frac{\chi}{\langle\rho\rangle}} .
$$

Due to (5) and the identity $\mathbf{N}_{1}(\varsigma) \mathbf{n}=-\mathbf{m}$ following from (1), the double and triple integrals (8) in (6) reduce to single integrals, involving contractions of the Stroh matrix block $\mathbf{N}_{3}(\varsigma)$ with the wave normal $\mathbf{m}$ and the eigenvectors 
$\overline{\mathbf{e}}_{\alpha}$ of $\left\langle\mathbf{N}_{3}\right\rangle$. Introduce the auxiliary notations

$$
\left\{\begin{array}{l}
g(\varsigma)=\mathbf{m} \cdot \mathbf{N}_{3}(\varsigma) \mathbf{m} \\
f_{\alpha}(\varsigma)=\mathbf{m} \cdot \mathbf{N}_{3}(\varsigma) \overline{\mathbf{e}}_{\alpha}
\end{array}, \quad\left\{\begin{array}{l}
g^{(j)}=\mathbf{m} \cdot \mathbf{N}_{3}^{(j)} \mathbf{m} \\
f_{\alpha}^{(j)}=\mathbf{m} \cdot \mathbf{N}_{3}^{(j)} \overline{\mathbf{e}}_{\alpha}
\end{array}, \quad\left\{\begin{array}{l}
\langle g\rangle=\mathbf{m} \cdot\left\langle\mathbf{N}_{3}\right\rangle \mathbf{m} \\
\langle f\rangle_{\alpha}=\mathbf{m} \cdot\left\langle\mathbf{N}_{3}\right\rangle \overline{\mathbf{e}}_{\alpha}
\end{array},\right.\right.\right.
$$

for which we note the identity

$$
g(\varsigma)=\sum_{\alpha=2,3} \frac{\left\langle f_{\alpha}\right\rangle}{\bar{\eta}_{\alpha}} f_{\alpha}(\varsigma), \quad g^{(j)}=\sum_{\alpha=2,3} \frac{\left\langle f_{\alpha}\right\rangle}{\bar{\eta}_{\alpha}} f_{\alpha}^{(j)}
$$

ensuing from (5). For an arbitrary $\mathbf{c}(\varsigma)$ and $\rho(\varsigma)$,

$$
\chi=\frac{1}{8} \int_{-1}^{1} \varsigma^{2} g(\varsigma) \mathrm{d} \varsigma-\sum_{\alpha=2,3} \frac{1}{16 \bar{\eta}_{\alpha}}\left(\int_{-1}^{1} \varsigma f_{\alpha}(\varsigma) \mathrm{d} \varsigma\right)^{2}
$$

If desirable, Eq. (12) may be presented as a sum of double integrals:

$$
\begin{aligned}
\chi & =\sum_{\alpha=2,3} \frac{1}{16 \bar{\eta}_{\alpha}}\left[\int_{-1}^{1} f_{\alpha}(\varsigma) \mathrm{d} \varsigma \int_{-1}^{1} \varsigma^{2} f_{\alpha}(\varsigma) \mathrm{d} \varsigma-\left(\int_{-1}^{1} \varsigma f_{\alpha}(\varsigma) \mathrm{d} \varsigma\right)^{2}\right] \\
& =\sum_{\alpha=2,3} \frac{1}{16 \bar{\eta}_{\alpha}}\left[\int_{-1}^{1} \int_{-1}^{\varsigma}\left(\varsigma-\varsigma_{1}\right)^{2} f_{\alpha}(\varsigma) f_{\alpha}\left(\varsigma_{1}\right) \mathrm{d} \varsigma \mathrm{d} \varsigma_{1}\right],
\end{aligned}
$$

where $\int_{-1}^{1} \int_{-1}^{\varsigma}=\frac{1}{2} \int_{-1}^{1} \int_{-1}^{1}$, and $\sum_{\alpha=2,3} \bar{\eta}_{\alpha}^{-1} f_{\alpha}(\varsigma) f_{\alpha}\left(\varsigma_{1}\right)=\mathbf{m} \cdot \mathbf{N}_{3}(\varsigma) \mathbf{J N} \mathbf{N}_{3}\left(\varsigma_{1}\right) \mathbf{m}$ with $\mathbf{J}=\sum_{\alpha=2,3} \bar{\eta}_{\alpha}^{-1} \overline{\mathbf{e}}_{\alpha} \otimes \overline{\mathbf{e}}_{\alpha}$ being the pseudo-inverse of $\left\langle\mathbf{N}_{3}\right\rangle$. For a discretely inhomogeneous plate consisting of $n$ homogeneous layers, Eq. (12) may be re-written as

$$
\chi=\sum_{j=1}^{n} g^{(j)} h_{j}\left(S_{j}^{2}+\frac{h_{j}^{2}}{12}\right)-\sum_{\alpha=2,3} \frac{1}{\bar{\eta}_{\alpha}}\left(\sum_{j=1}^{n} f_{\alpha}^{(j)} h_{j} S_{j}\right)^{2},
$$

with $S_{j}=-\frac{1}{2}\left(1+h_{j}\right)+\sum_{i=1}^{j} h_{i}$, which in the case of equidistant layers $\left(h_{j}=1 / n\right)$ is simply $S_{j}=(2 j-n-$ 1) $/ 2 n$ (using (11) enables further manipulation with (14)).

Let us relate the exact coefficient $\chi$ and slope $\kappa=\sqrt{\chi /\langle\rho\rangle}$, following from (12), to the 'would-be' estimate

$$
\chi^{(\mathrm{avrg})}=\frac{\langle g\rangle}{12}, \quad \kappa^{(\mathrm{avrg})}=\sqrt{\frac{\chi^{(\mathrm{avrg})}}{\langle\rho\rangle}},
$$

which is obtained by inserting the statically averaged $\langle\rho\rangle$ and $\left\langle\mathbf{N}_{3}\right\rangle$ into the corresponding formula for a homogeneous plate $v_{1}^{(\text {hom })}=2 k H \sqrt{g / 12 \rho}$ [9] (see (12) with constant $\left.\rho, g, f_{\alpha}\right)$. By $(12),(0<) \chi<3 \chi^{(\text {avrg) }}$ and so

$$
(0<) \kappa<\sqrt{3} \kappa^{(\mathrm{avrg})},
$$

i.e. the flexural branch slope $\kappa$ in a plate with an arbitrary profile of inhomogeneity is limited from above by $\sqrt{3} \kappa^{\text {(avrg) }}$. The upper bound (limit), implying an infinitely stiff and infinitely thin coating, is certainly not reachable.

Now consider the case of a periodically inhomogeneous plate, consisting of, say, $N$ periods $T=2 H / N$ within $[-H, H]$. For clarity, we resume the variable $y$ : e.g. $g(y)=\mathbf{m} \cdot \mathbf{N}_{3}(y) \mathbf{m}$ (see $(10)$ ) and $\langle\cdots\rangle=\frac{1}{T} \int_{0}^{T} \cdots(y) \mathrm{d} y$. 
Eq. (12) referred to the periodic setting may be arranged into the form

$$
\chi=\chi^{(\mathrm{avrg})}+\frac{1}{N^{2}}\left\{-\chi^{(\mathrm{avrg})}+\frac{1}{T^{3}}\left[\int_{0}^{T} y^{2} g(y) \mathrm{d} y-\sum_{\alpha=2,3} \frac{1}{T \bar{\eta}_{\alpha}}\left(\int_{0}^{T} y f_{\alpha}(y) \mathrm{d} y\right)^{2}\right]\right\},
$$

where the terms enclosed in brackets admit modification similarly to (13). By (17), the difference between the true value of the coefficient $\chi$ and the static averaging benchmark $\chi^{(\mathrm{avrg})}$ is proportional to $N^{-2}$. More specifically, its magnitude is of the order of $\left(\Delta_{T} / N\right)^{2}$ (not sign-definite though), where $\Delta_{T} \sim T \mathbf{N}_{3}^{\prime} /\left\langle\mathbf{N}_{3}\right\rangle$ or $\Delta_{T} \sim\left(\mathbf{N}_{3}^{(m)}-\right.$ $\left.\mathbf{N}_{3}^{(1)}\right) /\left\langle\mathbf{N}_{3}\right\rangle$ is the measure of variation of $\mathbf{N}_{3}(y)$ through a unit cell, continuously inhomogeneous or consisting of $m$ homogeneous layers, respectively. Thus, if the number of periods in a plate is large enough to make $\left(\Delta_{T} / N\right)^{2} \ll 1$, then the coefficient $\chi$ and the slope $\kappa$ of the flexural branch can be approximated by means of static averaging. For example, let the unit cell consist of two layers with the thickness-to-period ratio $\tau_{1}=2 H_{1} / T=h_{1} N$ and $\tau_{2}=1-\tau_{1}$, so that $\chi^{(\mathrm{avrg})}=\frac{1}{12}\left(g^{(1)} \tau_{1}+g^{(2)} \tau_{2}\right)$. In this case, Eq. (17) yields

$$
\chi=\chi^{(\mathrm{avrg})}-\frac{\tau_{1} \tau_{2}}{4 N^{2}}\left[\tau_{1} \tau_{2} \sum_{\alpha=2,3} \frac{\left(f_{\alpha}^{(1)}-f_{\alpha}^{(2)}\right)^{2}}{\bar{\eta}_{\alpha}}+\frac{2}{3}\left(\tau_{1}-\tau_{2}\right)\left(g^{(1)}-g^{(2)}\right)\right] .
$$

It is clear that the condition for validating the static averaging approximation with respect to strictly periodic inhomogeneity can be appropriately generalized for the inhomogeneity profiles, which are rapidly oscillating but with little variation of the envelope (the former feature on its own is certainly not sufficient).

Note in conclusion the formula for the flexural displacement with the leading-order dispersion term:

$$
\mathbf{A}_{1}(\varsigma)=C\left[\mathbf{n}-\mathrm{i} k H\left(\mathbf{m} \varsigma-\sum_{\alpha=2,3} \frac{\overline{\mathbf{e}}_{\alpha}}{2 \bar{\eta}_{\alpha}} \int_{-1}^{1} \varsigma f_{\alpha}(\varsigma) \mathrm{d} \varsigma\right)\right],
$$

where $C$ is a normalization constant. It is seen that inhomogeneity entails the 'out-of-plane' component (parallel to $\mathbf{m} \times \mathbf{n}$ ), unless it vanishes due to the appropriate material symmetry (see Section 3.2) or to the evenness of $f_{\alpha}(\varsigma)$.

\subsection{Symmetric and antisymmetric profiles of inhomogeneity}

For a generic profile of elastic inhomogeneity, the departure of $f_{\alpha}(\varsigma)$ from its mean value may be decomposed into two parts, even and odd with respect to the midplane:

$$
\begin{gathered}
f_{\alpha}(\varsigma)=\left\langle f_{\alpha}\right\rangle+\theta_{\alpha}(\varsigma), \quad \theta_{\alpha}(\varsigma)=\theta_{\alpha}^{(\text {even })}(\varsigma)+\theta_{\alpha}^{(\text {odd })}(\varsigma), \\
\theta_{\alpha}^{(\text {even })}(\varsigma)=\frac{1}{2}\left[\theta_{\alpha}(\varsigma)+\theta_{\alpha}(-\varsigma)\right]=\theta_{\alpha}^{(\text {even })}(-\varsigma), \\
\theta_{\alpha}^{(\text {odd })}(\varsigma)=\frac{1}{2}\left[\theta_{\alpha}(\varsigma)-\theta_{\alpha}(-\varsigma)\right]=-\theta_{\alpha}^{(\text {odd })}(-\varsigma),
\end{gathered}
$$

which enables writing Eq. (12) in the equivalent form as

$$
\chi=\chi^{(\mathrm{avrg})}+\sum_{\alpha=2,3} \frac{1}{4 \bar{\eta}_{\alpha}}\left[\left\langle f_{\alpha}\right\rangle \int_{0}^{1} \varsigma^{2} \theta_{\alpha}^{(\text {even })}(\varsigma) \mathrm{d} \varsigma-\left(\int_{0}^{1} \varsigma \theta_{\alpha}^{\text {(odd })}(\varsigma) \mathrm{d} \varsigma\right)^{2}\right] .
$$

We shall examine the cases, when $\theta_{\alpha}(\varsigma)=\theta_{\alpha}^{(\text {even) }}(\varsigma)$ (symmetric profile) and $\theta_{\alpha}(\varsigma)=\theta_{\alpha}^{\text {(odd) }}(\varsigma)$ (antisymmetric profile). 


\subsubsection{Symmetric profile}

Suppose that the plate is subject to the same processing on both sides, so that the variation of its elastic properties is even with respect to the midplane and hence

$$
f_{\alpha}(\varsigma)=f_{\alpha}(-\varsigma)(\Rightarrow g(\varsigma)=g(-\varsigma))
$$

Then, the second integral in (12) vanishes and, with reference to (15),

$$
\chi=\frac{1}{4} \int_{0}^{1} \varsigma^{2} g(\varsigma) \mathrm{d} \varsigma=\chi^{(\mathrm{avrg})}+\frac{1}{4} \int_{0}^{1}\left(\varsigma^{2}-\frac{1}{3}\right) g(\varsigma) \mathrm{d} \varsigma,
$$

which also follows from (21) with $\theta_{\alpha}^{\text {(odd) }}=0$ due to

$$
\sum_{\alpha=2,3} \frac{1}{\bar{\eta}_{\alpha}}\left\langle f_{\alpha}\right\rangle \int_{0}^{1} \varsigma^{2} \theta_{\alpha}^{\text {(even })}(\varsigma) \mathrm{d} \varsigma=\int_{0}^{1} \varsigma^{2}[g(\varsigma)-\langle g\rangle] \mathrm{d} \varsigma=\int_{0}^{1}\left(\varsigma^{2}-\frac{1}{3}\right) g(\varsigma) \mathrm{d} \varsigma .
$$

It is seen that the slope $\kappa=\sqrt{\chi /\langle\rho\rangle}$ being higher or lower than its static averaging benchmark $\kappa^{(\mathrm{avrg})}=\sqrt{\chi^{(\mathrm{avrg})} /\langle\rho\rangle}$ suggests that the plate is markedly stiffer near the faces than in the middle or vice versa, respectively. Let us further assume that the (even) function $g(\varsigma)$ is monotonic throughout the face-midplane range,

$$
\operatorname{sign} g^{\prime}(\varsigma) \gtrless 0 \text { for } \varsigma \in[-1,0] \Leftrightarrow \operatorname{sign} g^{\prime}(\varsigma) \lessgtr 0 \text { for } \varsigma \in[0,1],
$$

which is also a practically common situation. Integrating $(23)_{2}$ by parts and applying the mean-value theorem enables us to specify (16) for the case on hand as follows:

$$
\begin{aligned}
& \kappa^{(\mathrm{avrg})} \leq \kappa<\sqrt{3} \kappa^{(\mathrm{avrg})}, \text { if } g(\varsigma) \text { monotonically decreases towards the midplane; } \\
& (0<) \kappa \leq \kappa^{(\mathrm{avrg})} \text {, if } g(\varsigma) \text { monotonically increases towards the midplane, }
\end{aligned}
$$

where equality holds if and only if $g(\varsigma)=$ constant through the plate. The same statement applies to the discretely inhomogeneous plates, whose layers are stacked in a way even with respect to the midplane (when the second sum in (14) is zero). Thus, gauging $\kappa$ relatively to $\kappa^{(\mathrm{avrg})}$ can reveal certain features of the inhomogeneity profile.

To fix the ideas, consider a simple model of the symmetric profile, namely, a sandwich structure with the same homogeneous skin layers and a homogeneous core layer. Let us label their material parameters by the superscripts $j=1$, 2, namely, $g^{(1)}, \rho^{(1)}\left(=g^{(3)}, \rho^{(3)}\right)$ for the skin layers and $g^{(2)}, \rho^{(2)}$ for the core layer. The dimensionless thickness of each skin layer and of the core layer relatively to the aggregate plate thickness (see (3)) is $h_{1}$ and $h_{2}=1-2 h_{1}$, respectively. Invoking (12) and (15) yields

$$
\kappa=\sqrt{\frac{2 g^{(1)} h_{1}\left(4 h_{1}^{2}-6 h_{1}+3\right)+g^{(2)}\left(1-2 h_{1}\right)^{3}}{12\left[2 h_{1}\left(\rho_{1}-\rho_{2}\right)+\rho_{2}\right]}}, \quad \kappa^{(\mathrm{avrg})}=\sqrt{\frac{2 h_{1}\left(g^{(1)}-g^{(2)}\right)+g^{(2)}}{12\left[2 h_{1}\left(\rho_{1}-\rho_{2}\right)+\rho_{2}\right]}} .
$$

Plotting $\kappa$ and $\kappa^{(\mathrm{avrg})}$ as functions of $h_{1}$ represents their dependence on the sandwich composition, in other words, on the shape of (discrete) inhomogeneity profile. Fig. 1 shows the curves $\kappa\left(h_{1}\right)$ and $\kappa^{(\mathrm{avrg})}\left(h_{1}\right)$ for the materials listed in Table 1. (Without loss of generality and for illuminating the chief points, the numerical illustrations involve symmetric media satisfying $g^{(j)}=\eta_{3}^{(j)}$, see (32) and (34) below.) Each plot (a-c) in Fig. 1 is referred to a pair of materials and displays the curves for two different sandwich structures, which correspond to interchanging the given materials as substances $j=1,2$ for the skin and core layers. Obviously, the values $\kappa(0)=\sqrt{\frac{1}{12} g^{(2)}}$ and $\kappa\left(\frac{1}{2}\right)=\sqrt{\frac{1}{12} g^{(1)}}$ at the edge points $h_{1}=0, \frac{1}{2}$ imply the homogeneous limits, for which the whole plate consists of the core $(j=2)$ and of the skin $(j=1)$ material, respectively. By $(26), \operatorname{sign}\left(\kappa-\kappa^{(\mathrm{avrg})}\right)=\operatorname{sign}\left(g^{(1)}-g^{(2)}\right)$ and thus, in accordance with (25), $\kappa^{(\mathrm{avrg})}<\kappa<\sqrt{3} \kappa^{(\mathrm{avrg})}$ for any $h_{1} \neq 0, \frac{1}{2}$ if the skin layers are stiffer, and $\kappa<\kappa^{(\mathrm{avrg})}$ if otherwise. 

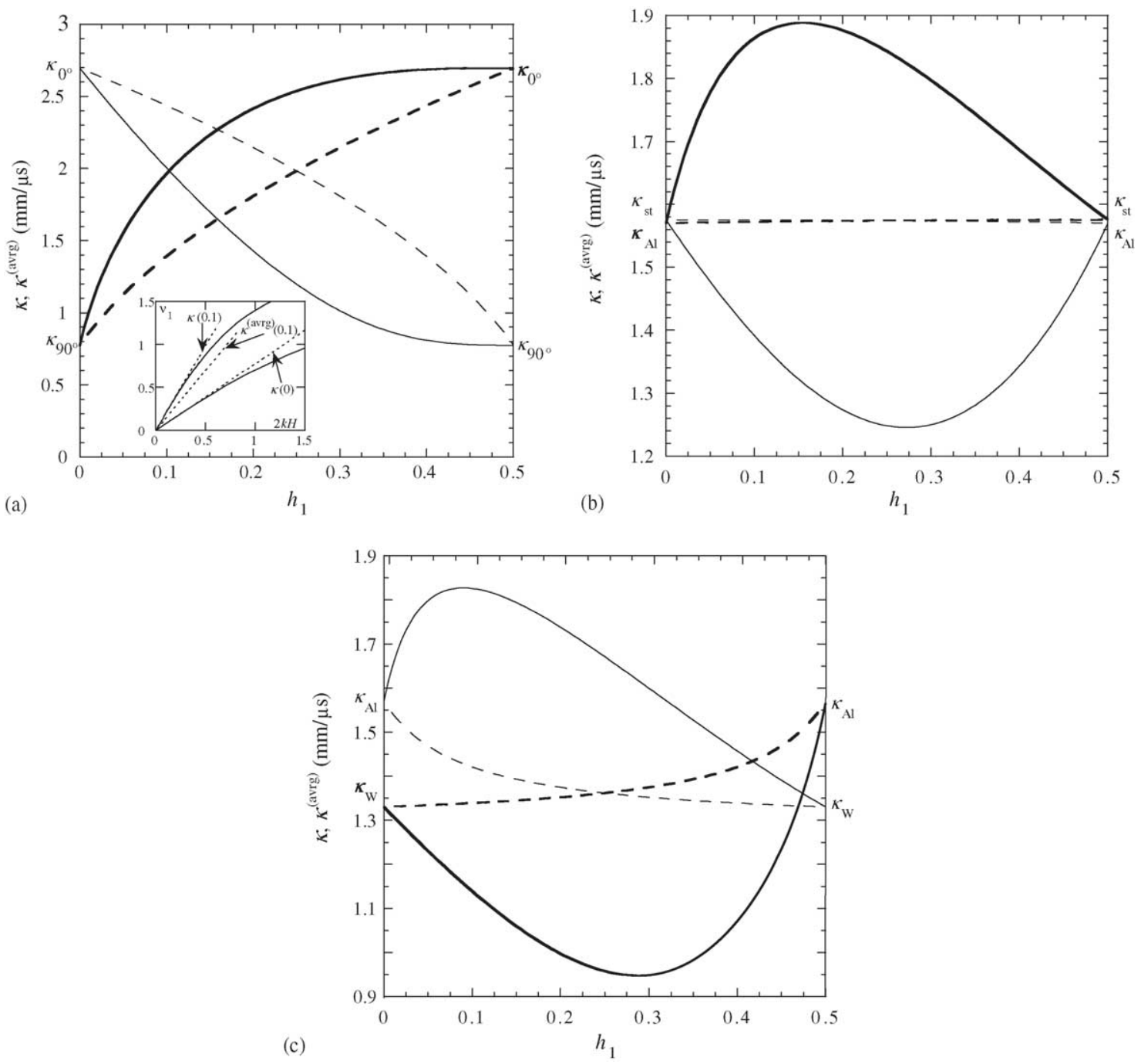

Fig. 1. The flexural branch slope $\kappa$ (solid lines) and the static averaging benchmark $\kappa^{(\mathrm{avrg})}$ (dashed lines) as functions of the dimensionless thickness $h_{1}$ of the skin layer in the sandwich structures made of cross-ply laminae (a); steel and aluminium (b); tungsten and aluminium (c). The material properties are listed in Table 1. For each pair of materials, the bold and thin lines correspond, respectively, to the 'fast'-'slow'-'fast' and 'slow'-'fast'-'slow' compositions of a sandwich (where 'fast' or 'slow' is understood in the sense of larger or lesser slope of the flexural branch in a homogeneous plate of each of the materials). The inset to (a) shows the flexural branch $v_{1}$ in the homogeneous plate of the 'slow' material (this branch slope is given by $\kappa(0)=\kappa_{90^{\circ}}$ ) and in the sandwich with 'fast' skin layers, occupying 0.1 of the sandwich thickness each (this branch slope is $\kappa(0.1)$, which is compared to $\kappa^{(\mathrm{arrg})}(0.1)$ ).

It is noted that varying relative thicknesses of the constituent layers changes the average density and thus brings the density distribution into play. In this regard, another aspect highlighted by Fig. 1 is a striking difference in the shape of curves $\kappa\left(h_{1}\right)$ for the two basically alternative options of choosing a pair of materials for a sandwich structure: when one of the materials is stiffer and lighter than the other, so that $g^{(1)} \gtrless g^{(2)}, \rho^{(1)} \lessgtr \rho^{(2)}$ (case (i)); when one of the materials is stiffer and heavier than the other, i.e. $g^{(1)} \gtrless g^{(2)}, \rho^{(1)} \gtrless \rho^{(2)}$ (case (ii)). The dependence $\kappa^{(\mathrm{avrg})}\left(h_{1}\right)$ is always monotonic for the both cases, whereas $\kappa\left(h_{1}\right)$ is monotonic in the case (i) (Fig. 1a) and is most likely 
Table 1

Properties of the materials involved in Figs. 1 and 3: the density $\rho\left(\mathrm{g} / \mathrm{cm}^{3}\right)$; the elastic constants $c_{I J}(\mathrm{GPa})$ for $X_{1} / / \mathbf{m}, X_{2} / / \mathbf{n}$; the stiffness coefficient $g=\eta_{3}(\mathrm{GPa})$; the slope of the flexural velocity branch $\kappa=\sqrt{\eta_{3} / 12 \rho}(\mathrm{mm} / \mu \mathrm{s})$, see (33) and (35)

\begin{tabular}{lccccr}
\hline & $\rho$ & $c_{11}$ & $c_{12}$ & $c_{22}$ & $\eta_{3}$ \\
\hline Aluminium, Al & 2.7 & 107.8 & 54.9 & $=c_{11}$ & 77.72 \\
Steel & 7.932 & 281.75 & 113.16 & $=c_{11}$ & 236.31 \\
Tungsten, W & 19.3 & 564.9 & 295.85 & $=c_{11}$ & 409.93 \\
Lamina [8, & 1.6 & & & 1.546 & 1.576 \\
$\quad 0^{\circ}$-orientation & & 143.2 & 7.5 & $=c_{11}$ & 1.330 \\
$90^{\circ}$-orientation & & 15.8 & 8.2 & 11.54 & 2.697 \\
\hline
\end{tabular}

to have an extremum in the case (ii) (Fig. $1 \mathrm{~b}$ and c). The point here is that, by contrast to $\kappa^{(\mathrm{avrg})}=\sqrt{\chi^{(\mathrm{avrg})} /\langle\rho\rangle}$, the flexural branch slope $\kappa=\sqrt{\chi /\langle\rho\rangle}$ is determined by the second-order momentum of the even stiffness profile (see $(23)_{1}$ ) along with the average of the density profile. The case (i) allows one material to be much 'faster' than the other, in the sense of markedly different flexural branch slopes for the homogeneous plates of each of the materials $j=1,2$. The contribution of momentum enhances the effect of relatively thin coating on the long-wave velocity of the flexural wave, accordingly to the through-plate distribution of the displacement field (19). For instance, Fig. 1a, engaging cross-plied laminae with strong elastic anisotropy ( $\rho$ is constant), shows that depositing the 'fast' skin layers, whose thickness is only 0.1 of the aggregate plate thickness, increases the flexural branch slope $\kappa$ by more than 2.5 times, while the static averaging benchmark $\kappa^{\text {(avrg) }}$ gives about $30 \%$ less than that. In turn, the case (ii) allows a pair of materials with quite different stiffnesses and densities to have similar values for the long-wave 'homogeneous' flexural velocity, which is when $g^{(1)} / \rho^{(1)}$ is close to $g^{(2)} / \rho^{(2)}$. Given that, the function $\kappa\left(h_{1}\right)$ is not monotonic and has one extremum within the interval $h_{1} \in\left(0, \frac{1}{2}\right)$. The difference between the curves $\kappa\left(h_{1}\right)$ and $\kappa^{(\mathrm{avrg})}\left(h_{1}\right)$ in Fig. $1 \mathrm{~b}$ and c pinpoints the aforementioned dissimilarity of how the stiffness and density distributions affect the flexural branch slope $\kappa$ in an inhomogeneous plate. Fig. $1 \mathrm{~b}$ exemplifies a sandwich structure, which is made of the materials with almost the same long-wave flexural velocity each $\left(\kappa(0) \approx \kappa\left(\frac{1}{2}\right)\right.$, hence $\kappa^{(\mathrm{avrg})}\left(h_{1}\right) \approx$ constant $)$, however, the velocity for the sandwich can be notably higher or lower than for the homogeneous plate of either of these materials. Fig. 1c demonstrates another interesting possibility within the case (ii). Here one of the materials is stiffer and at the same time 'slower' than the other material. As a result, according to (25), using the former 'slow' material as a coating for latter 'fast' one (hence $g^{(1)}>g^{(2)}, \kappa(0)<\kappa\left(\frac{1}{2}\right)$ ) yet makes the flexural velocity in the sandwich to be higher than in the homogeneous plate of the 'fast' core material (and vice versa for the inverse composition) - that is contrary to the case (i) (cf. Fig. 1a).

\subsubsection{Antisymmetric profile}

Another basic pattern of elastic inhomogeneity is its antisymmetric profile, in the sense that the departure of $f_{\alpha}(\varsigma)$ (and hence of $g(\varsigma)$ ) from the mean value is odd with respect to the midplane:

$$
f_{\alpha}(\varsigma)-\left\langle f_{\alpha}\right\rangle=-\left[f_{\alpha}(-\varsigma)-\left\langle f_{\alpha}\right\rangle\right]=\theta_{\alpha}^{(\text {odd })}(\varsigma),
$$

see (20). Under this condition, by (21),

$$
\chi=\chi^{(\mathrm{avrg})}-\sum_{\alpha=2,3} \frac{1}{4 \bar{\eta}_{\alpha}}\left[\int_{0}^{1} \varsigma \theta_{\alpha}^{(\mathrm{odd})}(\varsigma) \mathrm{d} \varsigma\right]^{2}\left(<\chi^{(\mathrm{avrg})}\right),
$$

and thus $\kappa<\kappa^{(\mathrm{avrg})}$. Moreover, if, in addition to (27), $f_{\alpha}(\varsigma) \geq 0$ for any $\varsigma \in[-1,1]$, which holds typically and is always the case for the monoclinic setting (32) (see Section 3.3), then $\chi>\frac{1}{4} \chi^{\text {(avrg) }}$ and so

$$
\frac{1}{2} \kappa^{(\mathrm{avrg})}<\kappa<\kappa^{(\mathrm{avrg})} .
$$




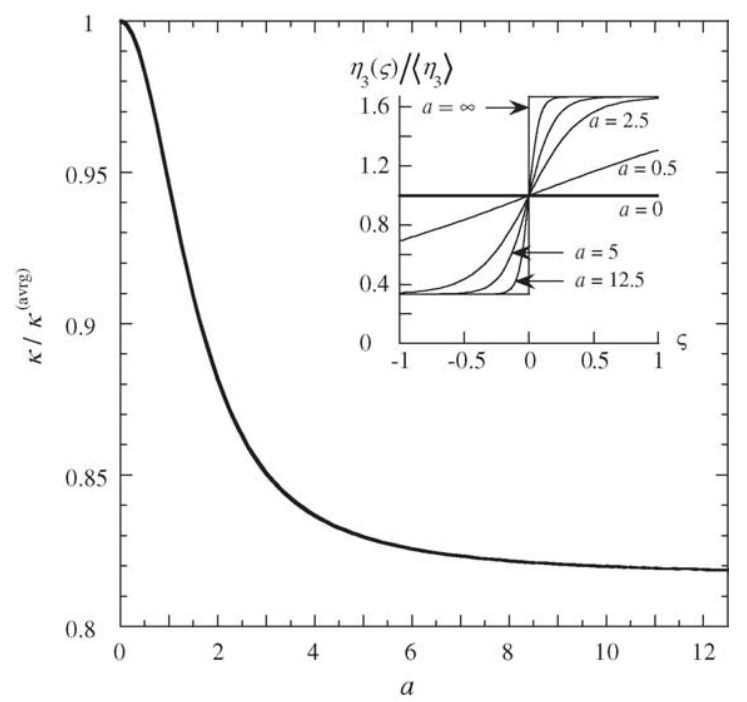

Fig. 2. The ratio $\kappa / \kappa^{(\mathrm{avrg})}$ as a function of the control parameter $a$ governing the antisymmetric profile shape (30) with a fixed $a_{0}=2 / 3$. The profile shapes are charted in the inset.

The lower bound $\frac{1}{2} \kappa^{(\mathrm{avrg})}$ is not approachable practically, for, it describes a theoretical limit when $\theta_{\alpha}^{(\text {(odd })}(\varsigma)$ is equal to $\left\langle f_{\alpha}\right\rangle$ for one-half of the plate and to $-\left\langle f_{\alpha}\right\rangle$ for the other half, which simply implies a homogeneous plate that is twice thinner than the given one and has the slope $\kappa^{(\mathrm{avrg})}$.

For exemplifying dependence on a variable shape of antisymmetric profile, it is pertinent to consider the case of continuous inhomogeneity, which may be viewed as a result of increasing external impact that has 'inverse signs' on the opposite plate faces. Suppose that this impact changes the antisymmetric profiles of stiffness and (possibly) density inhomogeneity without changing the mean, i.e. $\chi^{(\mathrm{avrg})},\langle\rho\rangle$ and $\kappa^{(\mathrm{avrg})}$ stay constant. The model example, displayed in Fig. 2, assumes the medium satisfying (32) and the stiffness profile described by

$$
\eta_{3}(\varsigma)=\left\langle\eta_{3}\right\rangle\left[1+a_{0} \tanh \left(a_{\varsigma}\right)\right]
$$

so that (28) yields

$$
\kappa=\kappa^{(\operatorname{avrg})} \sqrt{1-3\left[\int_{0}^{1} a_{0} \varsigma \tanh \left(a_{\varsigma}\right) \mathrm{d} \varsigma\right]^{2}} .
$$

\subsection{Simplification due to the vertical plane of material symmetry}

Consider a monoclinic plate with the symmetry plane $m$, which is either parallel to the sagittal plane $(\mathbf{m}, \mathbf{n})$ or orthogonal to the wave normal $\mathbf{m}$. Then, $\mathbf{m}$ and $\mathbf{t}=\mathbf{m} \times \mathbf{n}$ are the eigenvectors of $\mathbf{N}_{3}(\varsigma)$ at any $\varsigma$ and hence of $\left\langle\mathbf{N}_{3}\right\rangle$, so that $\mathbf{m} \cdot \mathbf{N}_{3}(\varsigma) \mathbf{t}=\mathbf{m} \cdot\left\langle\mathbf{N}_{3}\right\rangle \mathbf{t}=0$ and the eigenvalues $\bar{\eta}_{\alpha}$ of $\left\langle\mathbf{N}_{3}\right\rangle$ are equal to the statical average $\left\langle\eta_{\alpha}\right\rangle$ of the eigenvalues $\eta_{\alpha}(\varsigma)$ of $\mathbf{N}_{3}(\varsigma)$, see (5). Let, say, $\mathbf{t}=\mathbf{e}_{2}(\varsigma)=\overline{\mathbf{e}}_{2}, \mathbf{m}=\mathbf{e}_{3}(\varsigma)=\overline{\mathbf{e}}_{3}$. Thus, incorporating discrete inhomogeneity as well,

$$
\begin{aligned}
& f_{2}(\varsigma)=0, f_{3}(\varsigma)=g(\varsigma)=\eta_{3}(\varsigma), \quad \bar{\eta}_{3}=\left\langle\eta_{3}\right\rangle=\langle g\rangle, \\
& f_{2}^{(j)}=0, f_{3}^{(j)}=g^{(j)}=\eta_{3}^{(j)}
\end{aligned}
$$


where $\eta_{3}(\varsigma)$ or $\eta_{3}^{(j)}$ is

$$
\eta_{3}=\left\{\begin{array}{l}
c_{11}-\frac{c_{22} c_{16}^{2}-2 c_{12} c_{16} c_{26}+c_{12}^{2} c_{66}}{c_{22} c_{66}-c_{26}^{2}}, \text { if } \mathbf{m} \text { is parallel to } m, \\
c_{11}-\frac{c_{22} c_{14}^{2}-2 c_{12} c_{14} c_{24}+c_{12}^{2} c_{44}}{c_{22} c_{44}-c_{24}^{2}}, \text { if } \mathbf{m} \text { is orthogonal to } m,
\end{array}\right.
$$

given that the elastic coefficients $c_{I J}(\varsigma)$ or $c_{I J}^{(j)}$ are referred to the coordinate axes $X_{1}, X_{2}, X_{3}$ parallel to $\mathbf{m}, \mathbf{n}, \mathbf{t}$, respectively. Evidently, Eq. (33) reduces to

$$
\eta_{3}=\left\{\begin{array}{l}
c_{11}-c_{12}^{2} / c_{22}, \text { if the plate is orthorhombic, } \\
4 \rho v_{t}^{2}\left(1-v_{t}^{2} / v_{l}^{2}\right), \text { if }(\mathbf{m}, \mathbf{n}) \text { is the plane of isotropy }
\end{array}\right.
$$

$v_{l, t}$ being the longitudinal and transverse bulk-wave velocities.

Once (32) holds true, Eqs. (12) and (13) can be simplified as

$$
\chi=\frac{1}{8} \int_{-1}^{1} \varsigma^{2} \eta_{3}(\varsigma) \mathrm{d} \varsigma-\frac{1}{16\left\langle\eta_{3}\right\rangle}\left(\int_{-1}^{1} \varsigma \eta_{3}(\varsigma) \mathrm{d} \varsigma\right)^{2}=\frac{1}{16\left\langle\eta_{3}\right\rangle} \int_{-1}^{1} \int_{-1}^{\varsigma}\left(\varsigma-\varsigma_{1}\right)^{2} \eta_{3}(\varsigma) \eta_{3}\left(\varsigma_{1}\right) \mathrm{d} \varsigma \mathrm{d} \varsigma_{1} .
$$

Eq. (14), describing discretely inhomogeneous plate, reduces to

$$
\chi=\frac{1}{\left\langle\eta_{3}\right\rangle} \sum_{j=1}^{n} \sum_{k=1}^{j-1} \eta_{3}^{(j)} \eta_{3}^{(k)} h_{j} h_{k}\left(S_{j}-S_{k}\right)^{2}+\frac{1}{12} \sum_{j=1}^{n} \eta_{3}^{(j)} h_{j}^{3}
$$

which specifies for equidistant layers $\left(h_{j}=1 / n,\left\langle\eta_{3}\right\rangle=\frac{1}{n} \sum_{j=1}^{n} \eta_{3}^{(j)}\right)$ as

$$
\chi=\frac{1}{n^{4}\left\langle\eta_{3}\right\rangle} \sum_{j=1}^{n} \sum_{k=1}^{j-1} \eta_{3}^{(j)} \eta_{3}^{(k)}(j-k)^{2}+\frac{1}{12 n^{2}}\left\langle\eta_{3}\right\rangle
$$

Note that the first term on the right-hand sides of (36) and (37) is asymptotically independent of $n$, whereas the second term decreases as $n^{-2}$ with growing $n$. Eq. (17) for a periodic plate becomes

$$
\chi=\chi^{(\mathrm{avrg})}+\frac{1}{N^{2}}\left[-\chi^{(\mathrm{avrg})}+\frac{1}{T^{4}} \int_{0}^{T} \int_{0}^{y}\left(y_{1}-y\right)^{2} \eta_{3}(y) \eta_{3}\left(y_{1}\right) \mathrm{d} y \mathrm{~d} y_{1}\right] .
$$

Consider in some detail the particular case of a stack of $N$ identical monoclinic bilayers satisfying (32). The latter casts Eq. (18) into the form

$$
\chi=\chi^{(\mathrm{avrg})}-\frac{\tau_{1} \tau_{2}}{4 N^{2}}\left(\eta_{3}^{(1)}-\eta_{3}^{(2)}\right)\left[\tau_{1} \tau_{2} \frac{\eta_{3}^{(1)}-\eta_{3}^{(2)}}{\left\langle\eta_{3}\right\rangle}+\frac{2}{3}\left(\tau_{1}-\tau_{2}\right)\right],
$$

where $\chi^{(\mathrm{avrg})}=\frac{1}{12}\left(\eta_{3}^{(1)} \tau_{1}+\eta_{3}^{(2)} \tau_{2}\right)$ and $\tau_{2}=1-\tau_{1}$. Let us label parameters of the stiffer and softer layer by superscripts (1) and (2), so that $\eta_{3}^{(1)}>\eta_{3}^{(2)}$ (it does not matter indeed which of them is upper or lower in a periodic stack). If the stiffer layer is thicker than the softer layer: $\tau_{1}>\tau_{2}$, then, by virtue of (39), $\chi<\chi^{(\mathrm{avrg})}$ and so the flexural branch slope $\kappa$ is always lower than the static averaging benchmark $\kappa^{(\mathrm{avrg})}$, tending to it rapidly with growing $N$. If the stiffer layer is thinner: $\tau_{1}<\tau_{2}$, then $\kappa$ may be either less or greater than $\kappa^{\text {(avrg) }}$ depending on the certain 
balance between the thicknesses and the elastic parameters of the layers. It is set by the sign of the term in brackets in (39) and reads as follows:

$$
\kappa>\kappa^{(\mathrm{avrg})} \Leftrightarrow \tau_{1}<1 / B, \quad \kappa<\kappa^{(\mathrm{avrg})} \Leftrightarrow \tau_{1}>1 / B,
$$

where

$$
B=1+\frac{1}{4}\left[b-1+\sqrt{(b-1)^{2}+16 b}\right](>2), \text { with } b \equiv \eta_{3}^{(1)} / \eta_{3}^{(2)}(>1) .
$$

The threshold between the two opposite trends (40) is when $\tau_{1}=1 / B(<0.5)$. Then, $\chi=\chi^{(\mathrm{avrg})}$ and so the slope $\kappa$ stays equal to $\kappa^{(\mathrm{avrg})}$ regardless of $N$.
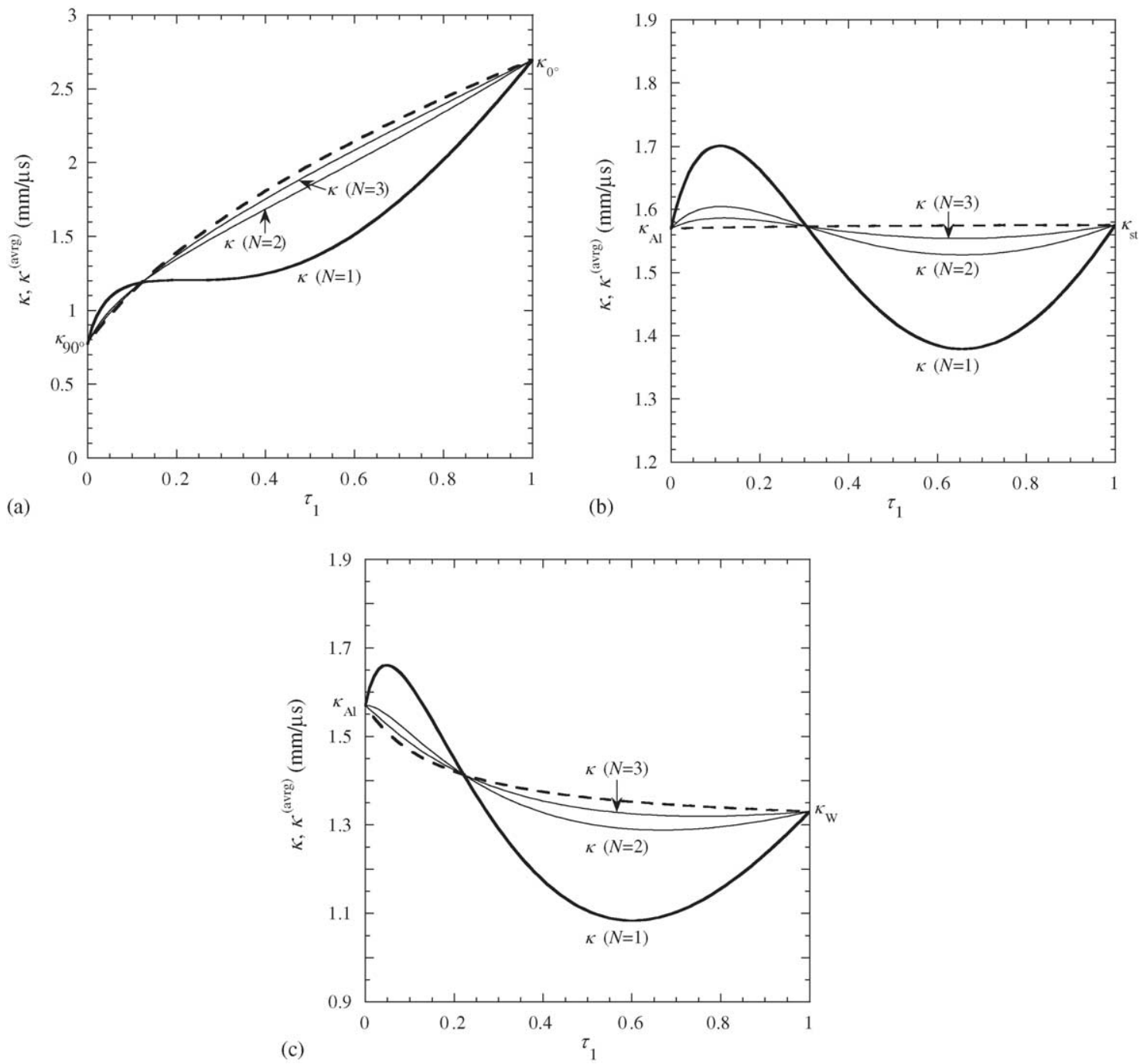

Fig. 3. The flexural branch slope $\kappa$ vs. the dimensionless thickness $\tau_{1}$ of the stiffer layer in a bilayered cell for the plate containing one cell (bold solid line), two cells and three cells (thin solid lines). The reference static averaging curve $\kappa^{(\mathrm{avrg})}\left(\tau_{1}\right)$ is plotted by dashed line. The bilayered cell is made of cross-ply laminae (a); steel and aluminium (b); tungsten and aluminium (c), characterized in Table 1. 
Fig. 3 shows the curves $\kappa\left(\tau_{1}\right)$ and $\kappa^{(\mathrm{avrg})}\left(\tau_{1}\right)$ for a plate with one, two and three bilayers $(N=1,2,3)$. The exemplified materials are the same as used in Fig. 1 for representing the cases (i) and (ii) defined in Section 3.2. The reference curve $\kappa^{(\mathrm{arrg})}\left(\tau_{1}\right)$ is always monotonic. The curve $\kappa\left(\tau_{1}\right)$, for any $N$, coincides with $\kappa^{(\mathrm{avrg})}\left(\tau_{1}\right)$ at the edge points $\tau_{1}=0,1$, which correspond to the homogeneous limits, and intersects $\kappa^{(\mathrm{avrg})}\left(\tau_{1}\right)$ at $\tau_{1}=1 / B$. Hence, $\kappa\left(\tau_{1}\right)$ may not have an extremum if $\kappa^{(\mathrm{avrg})}\left(\tau_{1}\right)$ is steep enough, which is when one of the layers is markedly 'faster' than the other (case (i), Fig. 3a), whereas $\kappa\left(\tau_{1}\right)$ has maximum at $\tau_{1}<1 / B$ and minimum at $\tau_{1}>1 / B$, if $\kappa^{(\mathrm{avrg})}\left(\tau_{1}\right)$ is flat enough (case (ii), Fig. 3b and c).

\section{4. $\mathrm{S}_{0}$ and $\mathrm{SH}_{0}$ branches}

The leading-order dispersion of the upper fundamental branches $\Delta v_{\alpha}(k)=v_{\alpha}(k)-v_{0 \alpha} \sim(2 k H)^{2}$ or $\Delta v_{\alpha}(\omega) \sim$ $\left(2 \omega H / v_{0 \alpha}\right)^{2}(\alpha=2,3)$ is somewhat less significant than that of the flexural branch $v_{1}(k)$. Departure of $v_{\alpha}(k)$ from $v_{0 \alpha}$ at $2 k H \ll 1$ is little, because the dispersion is quadratic and its coefficient is usually small. For inhomogeneous plates and general anisotropy, the coefficient of quadratic dispersion (7) can barely be simplified —it admits reducing triple integration to double only, and at the expense of sprawling terms. Certainly more tractable and actually more interesting is the specific case of the $\mathrm{SH}_{0}$ fundamental branch, whose dispersion is stipulated solely by the plate inhomogeneity. In view of these considerations, let us assume that the sagittal plane $(\mathbf{m}, \mathbf{n})$ coincides with a symmetry plane. One of the upper fundamental branches is the $\mathrm{SH}_{0}$ branch polarized along $\mathbf{t}=\mathbf{m} \times \mathbf{n}$. The conventional label $S_{0}$ ( $S$ for 'symmetric') is used for the other branch, in understanding though that it has attributes of a symmetric branch if and only if the horizontal symmetry plane, presumed in Section 4.1, is complemented by the symmetry (evenness) of inhomogeneity profile (see [10]). Keeping the notation $\mathbf{t}=\mathbf{e}_{2}(\varsigma)=\overline{\mathbf{e}}_{2}$ as in Section 3.2 specifies numbering of the branches $v_{\alpha}(k)$ as $v_{2}=v_{\mathrm{SH}_{0}}$ and $v_{3}=v_{S_{0}}$. In the subsequent formulas, the tensor $\mathbf{c}$ is referred to the same base as in (33) $\left(X_{1}, X_{2}, X_{3}\right.$ parallel to $\mathbf{m}, \mathbf{n}, \mathbf{t}$, respectively).

\section{1. $S_{0}$ branch}

For an orthorhombic inhomogeneous plate with symmetry planes parallel to the boundary (when $\overline{\mathbf{e}}_{\alpha}$. $\mathbf{P}_{3}^{(2)}\left(v_{0 \alpha}\right) \overline{\mathbf{e}}_{\beta}=0, \alpha, \beta=2,3$ in (7)) and to the sagittal plane, the $S_{0}$ velocity truncated at the leading-order dispersion term is defined by

$$
\begin{aligned}
\langle\rho\rangle v_{S_{0}}^{2}= & \left\langle\eta_{3}\right\rangle-(2 k H)^{2} \frac{1}{8}\left\{\int_{-1}^{1}\left[\int_{-1}^{\varsigma}\left(\varsigma-\varsigma_{1}\right) D\left(\varsigma_{1}\right) \mathrm{d} \varsigma_{1}-\int_{\varsigma}^{1}\left(\varsigma-\varsigma_{1}\right) B\left(\varsigma_{1}\right) \mathrm{d} \varsigma_{1}\right] C(\varsigma) \mathrm{d} \varsigma\right. \\
& \left.+\int_{-1}^{1}\left[\int_{-1}^{\varsigma} B\left(\varsigma_{1}\right) \mathrm{d} \varsigma_{1}\right]^{2} c_{66}^{-1}(\varsigma) \mathrm{d} \varsigma+\frac{\left\langle\eta_{3}\right\rangle}{2}\left[\int_{-1}^{1} C(\varsigma) \mathrm{d} \varsigma\right]^{2}-\frac{1}{2\left\langle\eta_{3}\right\rangle}\left[\int_{-1}^{1} \varsigma D(\varsigma) \mathrm{d} \varsigma\right]^{2}\right\},
\end{aligned}
$$

where

$$
D(\varsigma)=B-\left\langle\eta_{3}\right\rangle C, \quad B(\varsigma)=\eta_{3}-\left\langle\eta_{3}\right\rangle \frac{\rho}{\langle\rho\rangle}(\langle B\rangle=0), \quad C(\varsigma)=\frac{c_{12}}{c_{22}}, \quad \eta_{3}(\varsigma)=c_{11}-\frac{c_{12}^{2}}{c_{22}}
$$

with $\rho(\varsigma)$ and $c_{I J}(\varsigma)$. If the plate is periodic (hence $\langle\cdots\rangle=\frac{1}{T} \int_{0}^{T} \cdots \mathrm{d} y$ ), then with growing number $N$ of unit cells (42) tends at a rate $\sim N^{-2}$ to the static averaging approximation

$$
\langle\rho\rangle v_{S_{0}}^{(\mathrm{avrg}) 2}=\left\langle\eta_{3}\right\rangle\left[1-(2 k H)^{2} \frac{1}{12}\left\langle\frac{c_{12}}{c_{22}}\right\rangle^{2}\right],
$$

which is the exact formula for an 'effective' homogeneous plate with the Stroh matrix $\langle\mathbf{N}\rangle$, see [9]. 
For a simple case of a plate, consisting of two equidistant homogeneous layers $\left(h_{1}=h_{2}=\frac{1}{2}\right)$, it follows

$$
\begin{aligned}
\langle\rho\rangle v_{S_{0}}^{2}= & \left\langle\eta_{3}\right\rangle-(2 k H)^{2} \frac{1}{3 \times 2^{6}}\left\{5\left\langle\eta_{3}\right\rangle\left(C^{(1) 2}+\frac{6}{5} C^{(1)} C^{(2)}+C^{(2) 2}\right)-4\left(\frac{\rho^{(2)}}{\langle\rho\rangle} \eta_{3}^{(1)}-\frac{\rho^{(1)}}{\langle\rho\rangle} \eta_{3}^{(2)}\right)\right. \\
& \left.\times\left[C^{(1)}-C^{(2)}+\left(\frac{\rho^{(2)}}{\langle\rho\rangle} \eta_{3}^{(1)}-\frac{\rho^{(1)}}{\langle\rho\rangle} \eta_{3}^{(2)}\right)\left(\frac{3}{\left\langle\eta_{3}\right\rangle}-\frac{2}{c_{66}^{(1)}}-\frac{2}{c_{66}^{(2)}}\right)\right]\right\} .
\end{aligned}
$$

\section{2. $\mathrm{SH}_{0}$ branch}

Uncoupling of the SH motion due to the sagittal plane being a symmetry plane means that the SH waves are described by the $2 \times 2$ matricant (see, e.g. [14]). Vanishing of its left off-diagonal component gives the dispersion equation for the $\mathrm{SH}$ velocity branches in the form

$$
\langle\mu\rangle-\langle\rho\rangle v^{2}+\sum_{m=1}^{\infty}\left[p^{(2 m+1)}\left(v^{2}\right)\right](2 k H)^{2 m}=0,
$$

where $\mu=c_{55}-c_{45}^{2} / c_{44}$ and

$$
\begin{aligned}
p^{(2 m+1)}\left(v^{2}\right)= & \frac{1}{2^{2 m+1}} \int_{-1}^{1} \int_{-1}^{\varsigma} \cdots \int_{-1}^{\varsigma_{2 m-1}}\left[\mu(\varsigma)-\rho(\varsigma) v^{2}\right] c_{44}^{-1}\left(\varsigma_{1}\right)\left[\mu\left(\varsigma_{2}\right)-\rho\left(\varsigma_{2}\right) v^{2}\right] \\
& \times \cdots c_{44}^{-1}\left(\varsigma_{2 m-1}\right)\left[\mu\left(\varsigma_{2 m}\right)-\rho\left(\varsigma_{2 m}\right) v^{2}\right] \mathrm{d} \varsigma \mathrm{d} \varsigma_{1} \cdots \mathrm{d} \varsigma_{2 m} .
\end{aligned}
$$

The fundamental $\mathrm{SH}_{0}$ branch $v_{\mathrm{SH}_{0}}$ satisfies the series expansion

$$
\langle\rho\rangle v_{\mathrm{SH}_{0}}^{2}=\langle\mu\rangle+\sum_{m=1}^{\infty} x_{m}(2 k H)^{2 m},
$$

whose coefficients can be found recursively from (46) with (47). For instance,

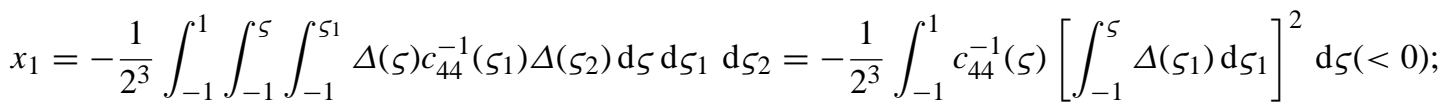

$$
\begin{aligned}
& x_{2}=\frac{1}{2^{5}} \int_{-1}^{1} \cdots \int_{-1}^{\varsigma_{3}} \Delta(\varsigma) c_{44}^{-1}\left(\varsigma_{1}\right) \Delta\left(\varsigma_{2}\right) c_{44}^{-1}\left(\varsigma_{3}\right) \Delta\left(\varsigma_{4}\right) \mathrm{d} \varsigma \cdots \mathrm{d} \varsigma_{4} \\
& -x_{1} \frac{1}{2^{3}} \int_{-1}^{1} \int_{-1}^{\varsigma} \int_{-1}^{\varsigma_{1}}\left[\rho(\varsigma) c_{44}^{-1}\left(\varsigma_{1}\right) \Delta\left(\varsigma_{2}\right)+\Delta(\varsigma) c_{44}^{-1}\left(\varsigma_{1}\right) \rho\left(\varsigma_{2}\right)\right] \mathrm{d} \varsigma \mathrm{d} \varsigma_{1} \mathrm{~d} \varsigma_{2} ; \text { etc., }
\end{aligned}
$$

where $\Delta(\varsigma)=\mu(\varsigma)-\langle\mu\rangle \rho(\varsigma) /\langle\rho\rangle$ (so $\langle\Delta\rangle=0$ ). Obviously, the dispersion of the $\mathrm{SH}_{0}$ branch encapsulates its departure from the static averaging, for, this branch is non-dispersive in a homogeneous plate.

A remarkable trait of the $\mathrm{SH}_{0}$ branch in a periodic plate with an arbitrary (monoclinic) continuously or discretely inhomogeneous unit cell is that this branch, along with a certain family of the upper SH branches, is independent of the number $N$ of periods $T$. It is merely a consequence of the $2 \times 2$ dimension of the 'SH matricant' (denote it, say, by $\mathbf{w}=\left\{w_{i j}\right\}$ ) and of the simple fact that an off-diagonal component $i j$ of arbitrary power of a $2 \times 2$ matrix is proportional to the same $i j$ th component of the matrix itself. Therefore, the dispersion equation $\left[\mathbf{w}^{N}(T, 0)\right]_{21}=0$ factors out the equation $w_{21}(T, 0)=0$, and so the spectrum $v_{\mathrm{SH}}(k T)$ for a plate with any number $N$ of unit cells with a fixed period $T$ includes $N$-independent family of branches (incorporating the fundamental, $\mathrm{SH}_{0}$ branch), which is 


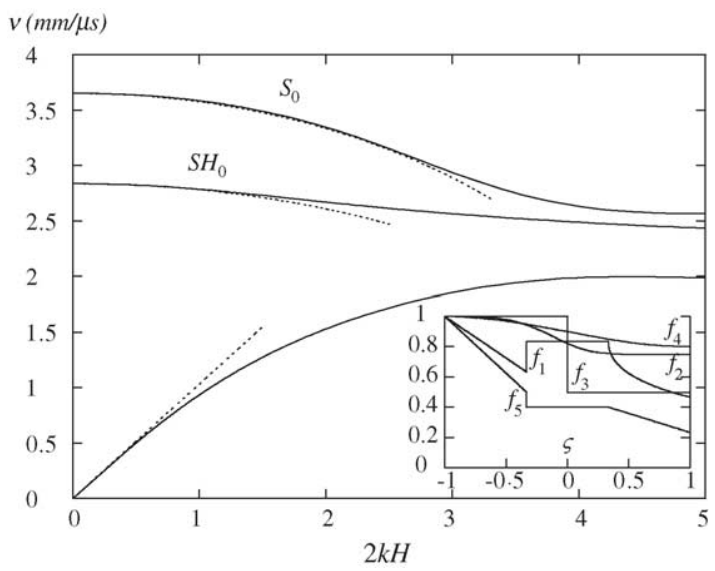

Fig. 4. The example explained in Section 5. Solid lines represent the exact numerical calculation; dotted lines show the analytical evaluation for the flexural branch slope (Eq. (35)) and for the leading-order dispersion of the $S_{0}$ (Eq. (42)) and the $\mathrm{SH}_{0}($ Eq. (49) 1 ) branches. The inset displays the profiles of material properties variation, see (50).

the intact spectrum for a single traction-free unit cell. Each of these branches corresponds to the Floquet mode with a complex wavenumber.

\section{Numerical example}

Fig. 4 shows numerically calculated fundamental branches within the long-wave domain and their leading-order analytical approximations for the orthorhombic inhomogeneous plate with the elastic coefficients and density chosen as follows:

$$
\begin{aligned}
& c_{11}=100 \times f_{1}(\varsigma), \quad c_{12}=50 \times f_{1}(\varsigma), \quad c_{66}=40 \times f_{1}(\varsigma), \quad c_{22}=75 \times f_{2}(\varsigma), \\
& c_{44}=10 \times f_{3}(\varsigma), \quad c_{55}=35 \times f_{4}(\varsigma)\left[\text { all in GPa] } ; \quad \rho=8 \times f_{5}(\varsigma) \mathrm{g} / \mathrm{cm}^{3} .\right.
\end{aligned}
$$

The functions $f_{1, \ldots, 5}(\varsigma)$, containing ranges of continuous variation (linear, cosine, exponential, Gaussian), constant plateaus and a stepwise discontinuity, are displayed in the inset to Fig. 4. The profiles are intentionally made up to be rather peculiar, merely for illustrating the potential of analytical and numerical means in handling arbitrary transverse inhomogeneity (see also [15]).

\section{Conclusions}

The coefficients of leading-order dispersion at the origin of the fundamental branches in a transversely inhomogeneous anisotropic plate have been studied. The most tractable is the flexural branch. The linear slope $\kappa$ of the flexural velocity branch $v_{1}(k)$ in a plate of unrestricted anisotropy is given by the simple formula with only single integrals of bilinear forms of the Stroh's matrix block, allowing further sizable simplification in the presence of a vertical plane of material symmetry parallel or orthogonal to the wave normal. The role of the static averaging has been elucidated. Given a generic inhomogeneity profile and arbitrary anisotropy, the upper limiting bound for $\kappa$ is $\sqrt{3}$ times the static averaging benchmark $\kappa^{(\mathrm{avrg})}$. If the profile of inhomogeneity is symmetric or antisymmetric with respect to the midplane, both the upper and the lower bounds for $\kappa$ can be further specified in terms of $\kappa^{(\mathrm{avrg})}$. 
A qualitative insight into some local features of the profile can be gained from comparing $\kappa$ and $\kappa^{(\mathrm{avrg})}$. This aspect is exemplified in detail for sandwich and bilayered structures. For the fundamental branches in periodic inhomogeneous plates, it is verified that the departure of the leading-order dispersion coefficients from the static averaging approximation is scaled by $\left(\Delta_{T} / N\right)^{2}$, where $\Delta_{T}$ is a measure of variation of the material properties through one period and $N$ is the number of periods in a plate. In this context, the exception is the fundamental branch of shear horizontal waves $\left(\mathrm{SH}_{0}\right.$ branch), which is altogether independent of the number of unit cells in any periodic plate regardless of the unit cell properties.

The results obtained for the fundamental branches in a traction-free plate can be engaged for other settings. For instance, the onset of the lowest real branch in a fluid-loaded inhomogeneous plate is related to the leadingorder term for the flexural branch of this plate free of traction in the same way as in the case of homogeneity: $v_{1}^{\text {(immersed) }}=v_{1}^{\text {(free) }} / \sqrt{1+\rho_{\mathrm{f}} /\langle\rho\rangle k H}$, where $v_{1}^{\text {(free) }}=2 \kappa k H$ and $\rho_{\mathrm{f}}$ is the fluid density. Another application, which is readily on hand, is concerned with the flexural modes propagating along the tip of a slender wedge, see [16]. Within the geometrical-acoustics approach, velocities of these wedge modes are expressed via the long-wave velocity of the plate flexural wave and thus can be estimated for a transversely inhomogeneous wedge.

\section{References}

[1] J.D. Achenbach, Wave propagation in fiber reinforced composites, in: G.P. Sendeckyi (Ed.), Composite Materials: Mechanics of Composite Materials, vol. 2, Academic Press, New York, 1974.

[2] A.K. Mal, T.C.T. Ting (Eds.), Wave Propagation in Struictural Composites, ASME-AMD, New York, 1988.

[3] A.H. Nayfeh, Wave Propagation in Layered Anisotropic Media, North-Holland, Amsterdam, 1995.

[4] D.E. Chimenti, Guided waves in plates and their use in material characterization, Appl. Mech. Rev. 50 (1997) 247-284.

[5] G.R. Liu, Z.C. Xi, Elastic Waves in Anisotropic Laminates, CRC Press, Boca Raton, FL, 2002.

[6] W.R. Rose, S.I. Rokhlin, L. Adler, Evaluation of anisotropic properties of graphite-epoxy composite plate using Lamb waves, in: D.O. Thompson, D.E. Chimenti (Eds.), Review of Progress in Quantitative Non-Destructive Evaluation, vol. 68, Plenum Press, New York, 1987, pp. 1111-1118.

[7] B. Hosten, Ultrasonic through-transmission for measuring complex stiffness moduli of composite materials, in: A.G. Every, W. Sachse (Eds.), Handbook of Elastic Properties of Solids, Liquids and Gases, vol. 1, Academic Press, San Diego, 2001, pp. 67-86 (Chapter 3).

[8] L. Wang, S.I. Rokhlin, Floquet wave homogenization of periodic anisotropic media, J. Acoust. Soc. Am. 112 (2002) 38-45;

L. Wang, S.I. Rokhlin, Floquet wave ultrasonic method for determination of single ply moduli in multilayered composites, J. Acoust. Soc. Am. 112 (2002) 916-924.

[9] A.L. Shuvalov, On the theory of wave propagation in anisotropic plates, Proc. R. Soc. Lond. A 456 (2000) 2197-2222.

[10] A.L. Shuvalov, O. Poncelet, M. Deschamps, General formalism for plane guided waves in transversely inhomogeneous anisotropic plates, Wave Motion 40 (2004) 413-426.

[11] V.I. Alshits, G. Maugin, Dynamics of multilayers: elastic waves in an anisotropic graded or stratified plate, Wave Motion 41 (2005) 357-394.

[12] M.C. Pease III, Methods of Matrix Algebra, Academic Press, New York, 1965.

[13] T.C.T. Ting, Anisotropic Elasticity, Oxford University Press, Oxford, 1996 (Chapter 7.2).

[14] K. Aki, P.G. Richards, Quantitative Seismology, Freeman and Co., San Franscisko, 1980.

[15] C. Baron, O. Poncelet, A. Shuvalov, Calculation of the velocity spectrum of the vertically inhomogeneous plates, in: Proceedings of 5th World Congress on Ultrasonics, September 7-10, Paris, France, 2003, pp. 605-608, available online at http://www.sfa.asso.fr/wcu2003/ procs/website/articles/000300.pdf.

[16] S.V. Birykov, Y.V. Gulyaev, V.V. Krylov, V.P. Plessky, Surface Acoustic Waves in Inhomogeneous Media, Springer, Berlin, 1995 (Chapter 10). 